\title{
An energy efficient and QoS aware routing protocol for wireless sensor and actuator networks
}

\author{
Souraya Yahiaoui ${ }^{1}$, Mawloud Omar ${ }^{1}$, Abdelmadjid Bouabdallah ${ }^{2}$ \\ Enrico Natalizio ${ }^{2}$, and Yacine Challal ${ }^{3}$ \\ ${ }^{1}$ Laboratoire d'Informatique Médicale, Faculté des Sciences Exactes \\ Université de Bejaia, 06000 Bejaia, Algérie. \\ ${ }^{2}$ Laboratoire Heudiasyc, Université de Technologie de Compiègne, 6599 Compiègne, France. \\ ${ }^{3}$ Ecole Supérieure d'Informatique, Alger, Algérie.
}

\begin{abstract}
Wireless Sensor and Actuator Networks (WSANs) are composed of a set of heterogeneous sensor and actuator nodes interconnected via wireless links. In WSANs, the actuators are responsible for taking prompt decisions and react accordingly to the data gathered by sensor nodes. In order to ensure efficient actions in such networks, we propose a new routing protocol that provides QoS in terms of end-to-end delay and energy consumption. The network is organized in clusters supervised by CHs (Cluster-Heads), elected according to a set of important metrics, namely the energy capability, the riches of connectivity, which is used to select the $\mathrm{CH}$ with high node density, and the accessibility degree regarding all the actuator nodes. The latter metric is the distance in number of hops of sensor nodes relative to the actuator nodes. This metric enhances more the network reliability by reducing the communication delay when alerting the actuator nodes, and hence, reducing the energy consumption. To reach efficiently the actuator nodes, we design a delay and energy sensitive routing protocol based on-demand routing approach. Our protocol incurs less end-to-end delay and is energy efficient. We perform an overall evaluation of our approach through simulations. The obtained results show out performance of our approach while providing effective gain in terms of end-to-end communication delay and energy consumption.
\end{abstract}

Keywords: WSAN, Routing, Clustering, QoS, Energy consumption, End-to-end delay

\section{Introduction}

Wireless Sensor Networks (WSNs) consist in a large number of distributed miniature sensor nodes composed of capture unit, data processing, and communication capacities. These nodes can communicate with each other and are capable 
to detect events and transmit the sensed information (temperature, humidity, motion detection, etc.) to the base station directly or through other intermediate nodes. The need of reaction in the event area has encouraged the emergence of actuator nodes, which have a further actuation unit that converts an electronic signal to a physical movement. Moreover, there is a need for a standardized way of obtaining information related to the physical sensors which are commonly found in networking equipment. Information such as the current value of the sensor, the current operational status, and the data units precision associated with the sensor [35]. WSN is a class of Low-Power and Lossy Networks (LLNs), in which the network nodes are constrained. LLN nodes operate with constraints on processing power, memory, and energy (battery power). Their interconnects are characterized by high loss rates, low data rates, and instability [36].

Wireless Sensor and Actuator Networks (WSANs) are an extension of WSNs [1. WSANs are used in various applications such as home automation, battlefield surveillance, networked robot, precision agriculture, animal control, and environmental monitoring [15, 18]. The actuator nodes have more communication capability compared to the sensor nodes and are responsible to take decisions and react in the event area according to the received data. To take advantage of the ability of the actuator nodes, an efficient cooperative communication is required. Generally, we find in the literature three levels of coordination, namely sensorsensor, sensor-actuator and actuator-actuator. The sensor-sensor coordination is executed to gather information in the surveillance field. The sensor-actuator coordination is executed to report the collected data from the sensor to the actuator nodes. Finally, the actuator-actuator coordination is executed to perform the appropriate action by negotiation with the other actuator nodes [15]. Thus, WSANs produce three important operations, namely the event-sensing, the decision-taking and the acting in the event area.

The coordination depends on the network architecture. In the semi-automated architecture, the coordination is ensured by the base station, which is responsible for monitoring and managing the overall network. With this architecture, the sensor nodes send the collected information to the base station controller that produces control commands and sends them to the actuator nodes to perform the appropriate actions. The coordination in the automated architecture is provided without the existence of a central controller [1, 15]. Our framework focuses on the last approach because the automated architecture is more efficient, in which the detected event is sent directly to the actuator nodes allowing more rapidity in reaction.

As in WSNs, it is critically important to save energy. Several protocols have been proposed to reduce the energy consumption and to maximize the network lifetime [19, 20, 21]. WSANs are mainly deployed for critical frameworks, where a short delay reaction to the event is highly required. This requirement is imposed 
by several types of applications, such as remote medical systems, fire detection systems, industrial monitoring applications, intruder tracking, as well by many other applications. In such systems, timely delivery of the sensed data plays a crucial role in order to react immediately with the appropriate action. Providing Quality of Service (QoS) requirements in terms of end-to-end delay is one of the most important target in such networks, since the actions are performed on the surveillance field immediately after the event occurs. In WSANs, optimizing end-to-end delay and energy consumption must be achieved in order to ensures QoS requirements, since routing decisions can impact the whole network. In this context, many open researches have been discussed in the literature (kindly, refer to [1]).

In this paper, we propose an energy efficient and QoS aware routing protocol for WSANs providing low delay and energy consumption. We organize the network in clusters, where each cluster is supervised by a Cluster-Head $(\mathrm{CH})$. We introduce a novel metric in the $\mathrm{CH}$ election process, which is the accessibility of the candidate sensor nodes regarding all the actuator nodes in the network. This metric represents the distance in terms of multi-hop between the candidate node and the actuators. Beside this important metric, the sensor nodes with high energy capability and riches in connectivity will be prioritized. The riches of connectivity represents the riches in terms of neighbor node number. The latter metrics improve the reliability of the network by reducing the communication delay when alerting the actuator nodes, and hence, reducing the energy consumption. We also address the sensor-actuator communication and we propose an on-demand routing-based data communication protocol, which allows to reach the actuator nodes with minimum delay and reduced energy consumption. We perform an overall evaluation of our approach with comparison to other concurrent approaches through simulations. The obtained results show out performance of our approach while providing effective gain in terms of communication delay and energy consumption.

The rest of the paper is organized as follows. In Section 2, we present the related works on clustering and routing for WSANs. In Section 3, we give the detailed description of our framework. In Section 4, we present the results of performance evaluation, and finally in Section 5, we conclude the paper.

\section{Related work}

In the literature, there have been many protocols proposed for WSNs that can not be directly applied in the context of WSANs due to the heterogeneous nature of the network. WSANs introduce several open research challenges [1, 15, 17]. In this section, we review some relevant routing solutions based on clustering in Section 2.1, and some relevant approaches that have addressed uniquely the 
routing aspect in Section 2.2. In Figure 1, we classify the reviewed solutions, and finally, we give in Section 2.3 an overall analysis, in which we highlight our contributions regarding the literature.

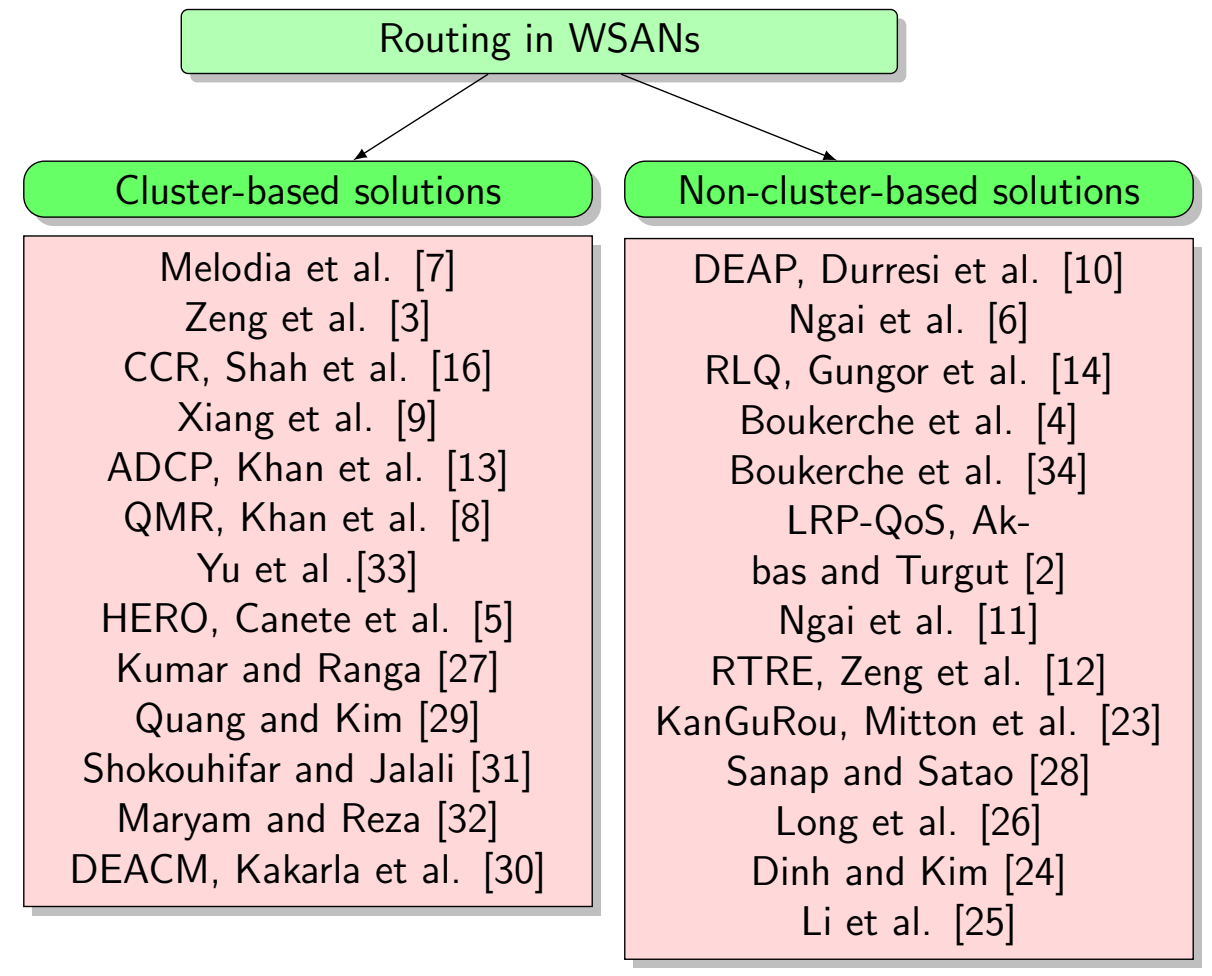

Figure 1: Classification of the existing solutions

\subsection{Cluster based routing solutions}

In [7], Melodia et al. have proposed a distributed coordination framework, which is an event-driven clustering paradigm. The clusters are created on-the-fly and only when necessary to provide reliability with low latency and minimum energy consumption. The sensor nodes coordinate with each other so as to optimally associate each sensor to an actuator node. Only the event area is clustered, and each cluster consists of those sensor nodes that send their data to the same actuator node. They have proposed a distributed energy-aware solution for sensor-actuator coordination aiming to minimize both the energy consumption and delay.

In [3], Zeng et al. have proposed a real-time architecture for automated WSANs. Each actuator node takes responsibility of collecting data from local sensor nodes by dividing the surveillance field into clusters. In each cluster, an actuator node acts as $\mathrm{CH}$ and the sensor nodes act as cluster members. When- 
ever, there is more than one event, the actuator node responds to the first detected event, which increases time response for the other events.

In [16], Shah et al. have proposed CCR (Cluster-based Coordination and Routing protocol), an integrated approach for WSANs that addresses the reliability and the energy efficiency in coordination between the sensor and actuator nodes. The sensor nodes form the clusters basing on the energy states and the network density. Afterwards, each $\mathrm{CH}$ coordinates with the actuator nodes to form an interactive region. The reliability is addressed in terms of data delivery time to the actuator nodes. They have also integrated a real-time data aggregation solution aiming to conserve the energy consumption by reducing the transmission overhead.

In [9], Xiang et al. have proposed a QoS architecture for automated WSANs, in which the zone of interest is organized into clusters and the actuator nodes act as CHs. They have proposed a sensor-actuator event reporting algorithm considering the delay, the energy and the load of actuator nodes. They have also presented a self-aware sensor-actuator coordination requirement mechanism in order to select the actuator node nearby for timely response, and an actuatoractuator negotiation algorithm to make ordering task assignment for multiple events with different priorities.

In [13], Khan et al. have proposed ADCP (Actor Directed Clustering Proto$\mathrm{col}$ ), in which the $\mathrm{CH}$ election depends on the actuator node locations in the area as well as the energy states of the candidate sensor nodes. A sensor node will be elected as a $\mathrm{CH}$, which has a lower distance with an actuator node as well as maximum energy in that cluster. After the cluster formation, the data communication is performed hop-by-hop to the actuator node by satisfying the energy level up to a pre-defined threshold and also directly accessible to it. If the energy of a nearest sensor node towards an actuator node is lower than the threshold, then the data packet will be transmitted to the next nearest one satisfying the energy condition.

In [8], Khan et al. have proposed the protocol QMR (QoS Multicast Routing), which handles the multicast routing using a clustering algorithm. The $\mathrm{CH}$ election is based only on the distance of the nearest actuator node to a given candidate sensor node. They have supposed that initially each sensor node is bound to a predefined single actuator node to report the event data through its $\mathrm{CH}$, which is transmitted to multiple CHs as well as actuator nodes.

In [33], Yu et al. have proposed a cluster-based routing protocol with nonuniform node distribution. A cluster of event sizes is constructed using a competition range. The $\mathrm{CHs}$ choose the nodes with higher energy and fewer member nodes as their next hops to communicate with the base station. In intra-cluster communication, the cluster members communicate with the $\mathrm{CHs}$ directly.

In [5], Canete et al. have proposed the protocol HERO (Hierarchical, Efficient 
and Reliable rOuting). The sensor nodes are supposed to be grouped in clusters and the collected data is transmitted to the $\mathrm{CH}$ (actuator and/or sink). In this protocol, the sensor nodes can join the clusters through their neighbors. The sensor nodes communicate the data to the $\mathrm{CH}$ using the shortest path with respect of the energy consumption balance. In order to establish the communication between the $\mathrm{CH}$ and the sensor nodes, they have introduced the concept of "clue node", in which a $\mathrm{CH}$ is able to estimate the area, where the sensor nodes are located.

In [27], Kumar and Ranga have proposed a cluster-based coordination and communication framework. The protocol is based on genetic algorithm with multitier clustering technique to transmit the data to the sink node using the actuator nodes. The clustering is formed on the sensor nodes, which will report the data to the nearest $\mathrm{CH}$. The $\mathrm{CH}$ may be chosen on the basis of energy parameters. The protocol use genetic algorithm to select $\mathrm{CH}$ both at sensor level as well as actuator level.

In [29], Quang and Kim have proposed a clustering algorithm for WSANs. A multi-level hierarchical structure can be applied and an heuristic path searching algorithm has been proposed to select the intermediate nodes. Then, a channel assignment scheme for sub-clusters is proposed to reduce the interference between the neighboring sub-clusters.

In [31], Shokouhifar and Jalali have proposed ASLPR (hybrid clustering-based Application-Specific Low Power Routing protocol) based on LEACH with an extension to the energy predication. ASLPR considers the distance from the sensors to the base station, the residual energy of the sensors, and the distance between the $\mathrm{CHs}$ in the election process. To optimize the controllable parameters of ASLPR, a hybrid algorithm based on genetic algorithm and simulated annealing is used.

In [32], Maryam and Reza have proposed a decentralized hierarchical clusterbased routing algorithm. The $\mathrm{CHs}$ are selected at the routing tree edges based on effective local information such as each node residual energy, its distance to the base station and the proximity to its neighbors. The base station is located at the root and the cluster member nodes in leaves.

In [30], Kakarla et al. have proposed the protocol DEACM (Delay and Energy Aware Coordination Mechanism). In DEACM, a two-level hierarchical k-hop clustering mechanism is used to organize the sensors and actuators for communication. In the first level, sensors form a k-hop cluster using actuators as CHs, and the sink is made as the $\mathrm{CH}$ in the second level to form a cluster among actuators.

\subsection{Non-clustering routing solutions}

In [10], Durresi et al. have proposed DEAP (Delay-Energy Aware Routing Protocol); an energy management scheme that applies duty cycle for energy conservation, and a loose geographical greedy routing protocol that in each hop dis- 
tributes the load among a group of neighboring nodes. It uses the advantage of actuator nodes resources to reduce the energy consumption of the others sensor nodes. The protocol is a distributed by a randomized algorithm, where the nodes make local decisions on whether to sleep or to be active.

In [6], Ngai et al. have proposed a real-time communication framework that supports the timely communication, providing low latency and coordination among the sensor and actuator nodes. The actuators diffuse periodically their positioning information to the adjacent sensor nodes. The protocol consists of two steps. The first is an algorithm of real-time and distributed event reporting for the sensor nodes to send the data to the actuator nodes over delay-sensitive routing paths. The data with more importance is transmitted with high priority. The second is an algorithm of coordination for actuator node selection basing on reaction-delay.

In [14], Gungor et al. have proposed RLQ (Resource-aware and Link Quality) based routing metric for WSANs. The RLQ metric is a combined link cost metric, which is based on energy efficiency and link quality statistics. Aiming to exploit the heterogeneous capabilities in WSANs, the protocol adapts to the varying wireless channel condition. The RLQ metric gives priority to the use of resourcerich actuator nodes instead of energy-constrained sensor nodes for packet routing and processing.

In [4, 34], Boukerche et al. have proposed an approach of service differentiation and central processing of routes aiming at low latency and reliable delivery in the presence of failures. Moreover, they have integrated to this approach an energyaware event-ordering mechanism. The main purpose is to interpret the right context from the different sensed data and then take the adequate decision. In this way, the actuator nodes are used to aggregate the time-correlated events from the sensor nodes and eliminate ambiguities.

In [2], Akbas and Turgut have proposed LRP-QoS (Lightweight Routing Protocol with QoS support), which differentiates the actions based on the sensed information from the sensor nodes, as well as using a dynamic packet tagging at the sensor nodes, and per flow management at actuator nodes. The packets are processed according to their importance. The criteria taken in charge are the rate of the flows and the transmission bandwidth.

In [11], Ngai et al. have proposed a centric approach, which combines an event data aggregation algorithm, a delay-aware priority based transmission protocol, and an adaptive actuator allocation algorithm. The routing process adopts smart priority scheduling that differentiates event data of non-uniform importance. They have extended the protocol to handle the node and link failures using an adaptive replication algorithm.

In [12], Zeng et al. have proposed RTRE (Real-Time data Report and task Execution), which takes in charge the delay, the energy and the reliability. They have proposed a self-organized sensor-actuator coordination scheme to support 
real-time event data collection, and a real-time scheme to make effective reactions to the sensed information. The latter operation is performed through task assignment negotiations in dynamic coordination among the actuator nodes.

In [23], Mitton et al. have proposed KanGuRou (k-ANycast GUaranteed delivery ROUting protocol), which is a reliability-aware multi-sink routing approach. In k-anycasting, a sensor node aiming to report event information, sends the data to any $k$ sinks in the network (sinks are acting as actuator nodes). They have introduced this aspect in order to increase the reliability of data transmission. The protocol constructs an energy-aware spanning tree reaching $k$ sinks among all the existing ones. To increase the reliability, a data packet is forwarded to $k$ sinks. At each step, the current sensor node computes a spanning tree over $k$ sinks with minimal cost. A sensor node running the protocol first computes a tree, including $k$ sinks with minimal weights. If this tree has more than one edge originated at the node itself, it duplicates the message following the edge number.

In [28], Sanap et Satao have proposed an algorithm for real-life applications. From the physical observation, the sensor nodes collect the information, which is forwarded to the actuator node and then, the set of actuator nodes send the data to the base station. The actuators in the network control the set of sensor nodes, and it would intimate them to desactivate during their non-busy hours.

In [26], Long et al. have proposed a disjoint multi-path routing scheme. The latter operates in two distinct stages. In the initial stage, each sensor node obtains the shortest hop to each actuator, and in the second stage is performed the multi-path routing. The source nodes communicate with the destination nodes by creating multiple routing paths among the actuator sets.

In [24], Dinh and Kim have proposed ADA (Actor-oriented Directional Anycast routing protocol), which supports arbitrary traffic. ADA exploits the directional antennas and actuators capabilities to route the data information to the base station, which takes the appropriate decisions. ADA exploits the actuators as main routing anchors to reduce traffic load for sensor nodes.

In [25], Li et al. have proposed an asymmetric routing approach for WSANs. Under asymmetric routing, the sensing and actuation routes can be configured independently from each other. The proposed protocol employs different routing strategies for sensing and actuation. This routing protocol employs source routing for sensing and graph routing for actuation.

\subsection{Overall analysis and our contributions}

Despite the availability of various solutions, there still exist limitations. Most existing solutions have only as main goal electing the best sensor node, which will act as a $\mathrm{CH}$ as long as possible. In this way, there exist various solutions with a mixture of weighted parameters, such as the energy, the riches of connectivity, etc. The main drawback of the existing solutions is the absence of parameters 
considering the accessibility degree of the candidate sensor nodes regarding the actuator nodes. The efficiency to reach the actuator nodes is an important criterion when designing solutions in WSANs context, which allows delay-efficiency in data reporting. In the $\mathrm{CH}$ election, few solutions select the nearest candidate node regarding an actuator without considering the number and positions of the other ones. By addressing these important aspects, our contribution is double:

1. In clustering point of view, we introduce a novel metric in the $\mathrm{CH}$ election, which is the accessibility degree in terms of multi-hop distance of the candidate sensor nodes regarding all the actuator nodes. Instead favoriting the nearest sensor to the actuator node, we give priority to the candidate sensor node having access to several actuator nodes, where its best position is in the center of actuators locations. This process of $\mathrm{CH}$ election is the main building-block of the routing approach, which represents the second part of our contribution.

2. After the $\mathrm{CH}$ election, we address the sensor-actuator communication and we propose an on-demand routing-based data communication protocol, which allows reaching the actuator node with minimum delay and low energy consumption. The proposed protocol integrates to the RREP (Route REPly) packet the energy state of intermediate sensor nodes and the response delay regarding the already transmitted RREQ (Route REQuest) packet. Thus, the source node is able to select the most efficient routing path with respect of both energy and delay when it receives the different RREQs.

\section{The proposed framework}

In this section, we present the network model, and then the detailed description of the proposed solutions in terms of clustering and routing.

\subsection{Network model}

The network that we consider is composed of a set of sensor and actuator nodes, which are interconnected through wireless links. We suppose that the actuator nodes are deployed uniformly to act homogeneously in the surveillance field. The actuator nodes are able to communicate with each other as well as with the sensor nodes. As in WSNs, the sensor nodes are small, static and resource-limited devices for monitoring the environment and reporting events to the actuator nodes. We assume that the actuator nodes are static devices having resource capability constraints, but more rich and with a longer range of transmission compared to the sensor nodes. The sensor nodes are organized in clusters, and for each cluster one sensor node is elected to act as a $\mathrm{CH}$. The cluster membership does not change over the time since the sensor nodes are static and the clustering is 
performed among the sensor nodes, which are geographically adjacent. In Figure 2, we illustrate the network model.

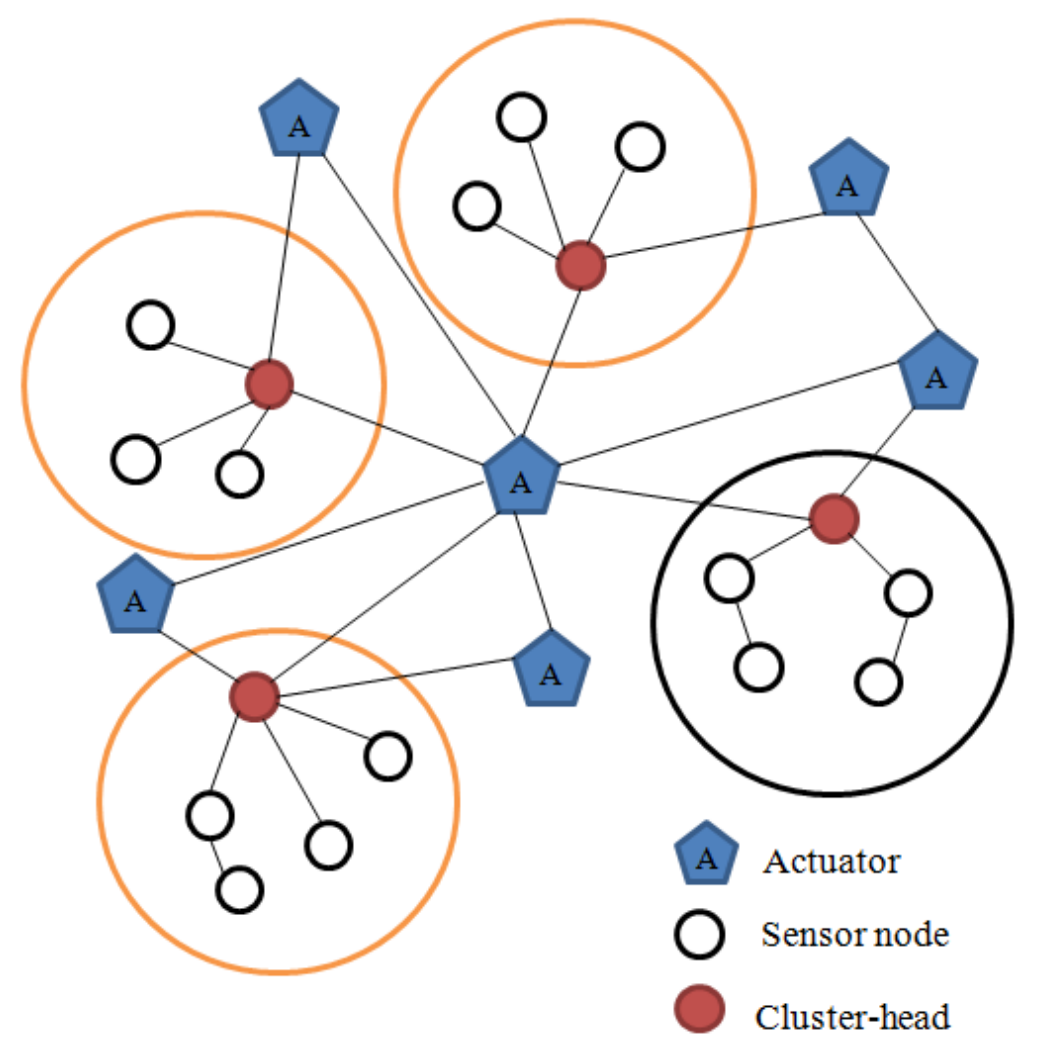

Figure 2: Network model

When a sensor node detects an event in the physical world, it gathers the data and sends it to its corresponding $\mathrm{CH}$. Afterwards, the latter node sends an alert to the best actuator node, which should react immediately to the event by performing the appropriate action. We notice that the $\mathrm{CH}$ is able to communicate with all the actuator nodes, which are in its region. We illustrate in Figure 3 , the communication model among the sensor, $\mathrm{CH}$ and actuator nodes. Table 1 presents a description of the used notations.

The proposed approach is designed for static WASNs, which is quite adapted for potential application fields, such as fire sprinkler systems monitoring, supervisory control and data acquisition system, industrial applications, as well for many other applications. However, in the context of mobile environments, a fully distributed cluster formation protocol should be designed in order maintain the cluster memberships despite the high frequency of nodes joining and departure. Moreover, a specific solution should be designed to guess and track the mobile actuator positions. In the scope of this paper, we do not address the mobility 


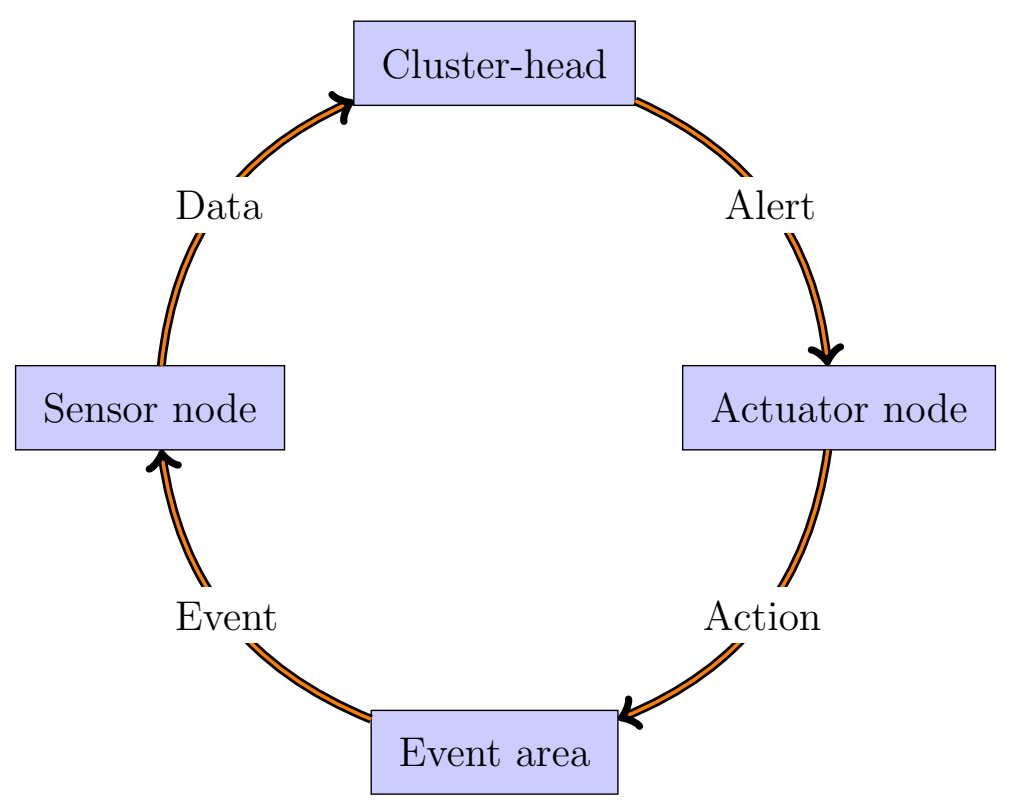

Figure 3: Communication model

aspect.

\begin{tabular}{|c|c|}
\hline Symbol & Description \\
\hline c & Coefficient \\
\hline$E_{i}$ & Residual energy state of the sensor node $i$ \\
\hline $\mathrm{D}_{\mathrm{i}}$ & Average distance of the sensor node $i$ to its neighbors \\
\hline $\mathrm{N}_{\mathrm{i}}$ & Number of the sensor node $i$ neighbors \\
\hline$H\left(i, a_{j}\right)$ & Multi-hop distance between the sensor node $i$ and the actuator node $j$ \\
\hline k & The number of actuator nodes in the same cluster \\
\hline $\mathrm{n}$ & The number of sensor nodes in the same cluster \\
\hline$W_{\text {th }}$ & Threshold weight \\
\hline $\mathrm{CM}$ & Liste of cluster members \\
\hline $\mathrm{P}\langle\mathrm{CH}, \mathrm{A}\rangle$ & Routing path from the $\mathrm{CH}$ to the actuator node $\mathrm{A}$ \\
\hline $\mathrm{C}(\mathrm{P}\langle\mathrm{CH}, \mathrm{A}\rangle)$ & Routing path $\mathrm{P}\langle\mathrm{CH}, \mathrm{A}\rangle$ cost \\
\hline $\mathrm{R}(\mathrm{P}\langle\mathrm{CH}, \mathrm{A}\rangle)$ & Routing path $\mathrm{P}\langle\mathrm{CH}, A\rangle$ average energy \\
\hline$\triangle(\mathrm{P}\langle\mathrm{CH}, \mathrm{A}\rangle)$ & Routing path $\mathrm{P}\langle\mathrm{CH}, \mathrm{A}\rangle$ delay \\
\hline
\end{tabular}

Table 1: Notations

\subsection{Delay-energy aware clustering algorithm}

The formation of clusters is performed at the moment of the network deployment. In our work, we do not consider a predetermined cluster formation before 
starting operation of the WSAN system. The deployment is performed randomly, where the number of nodes in each cluster is not fixed and the nodes membership to clusters depend on their initial geographical positions. Each node in the cluster is aware of all the cluster members, denoted by CM. To act accurately, we propose a $\mathrm{CH}$ election process by minimizing the communication delay and the energy consumption. To achieve this, each sensor node $i$ of the same cluster computes its weight $W_{i}$ based on its residual energy state, the density of its neighboring nodes, and the distances, which separate it to the actuator nodes. Our approach uses beaconing in order to capture these information. The beacon messages are exchanged between cluster members at each period of time $\gamma$ to establish and maintain the $\mathrm{CH}$ election. Each node broadcasts a beacon message to notify its presence to the cluster members. A beacon message contains all the states of the sensor nodes. Upon received, the weight $W_{i}$ is computed as follows:

$$
W_{i}=\frac{N_{i} \cdot c \cdot E_{i}}{D_{i}}+\frac{(1-c) \cdot k}{\sum_{j=1}^{k} H\left(i, a_{j}\right)} .
$$

The parameter $E_{i}$ denotes the sensor node $i$ residual energy state, which should be maximized in order to enhance the network lifetime. The parameter $D_{i}$ denotes the average distance of the sensor node $i$ to its neighbors, and $N_{i}$ represents the number of its neighbors. The parameter $D_{i}$ should be minimized and $N_{i}$ should be maximized in order to give priority to the riches of connectivity. The parameter $k$ denotes the number of actuator nodes on the cluster and $\sum_{j=1}^{k} H\left(i, a_{j}\right)$ denotes the sum of the multi-hop distances between the sensor node $i$ and all the actuator nodes. The first parameter should be maximized in order to give priority to the riches of accessibility to the actuator nodes, and the second one should be minimized to give priority to the delay of communication with the actuator nodes. The coefficient $c \in[0,1]$ should be adjusted in function of the application domain. For example, in the case of industrial applications, the data are typically time sensitive. Hence, it should be attributed a low value to $\mathrm{c}$ in order to elect a $\mathrm{CH}$ that is closest to the actuator nodes. Otherwise, if the value of $c$ is increased, we give more priority to the energy efficiency and riches of connectivity.

Upon computing the weight $W_{i}$, the sensor node $i$ compares it to a predefined threshold, denoted by $W_{t h}$. If $W_{i}>W_{t h}$, then the sensor node $i$ forwards a beacon message that contains the weight information to its neighbors. Upon receiving the beacon messages, each sensor node saves these information in its local storage. Since each sensor node in the cluster is aware of the weights of all the other cluster members, a sensor node with the higher weight is auto-elected as a $\mathrm{CH}$. In this way, each sensor node orders the weights. The weight $W_{n}$ being on the end of the queue represents the most appropriate candidate sensor node. Periodically, the $\mathrm{CH}$ checks its own weight $W_{n}$. Whenever $W_{n}$ is inferior of the 
previous weight $W_{n-1}$, then, the sensor node of weight $W_{n-1}$ is auto-elected as a $\mathrm{CH}$. If a weight is inferior than the threshold $W_{t h}$, it will be deleted from the queue. Algorithm 1 illustrates the pseudo-code of the $\mathrm{CH}$ election.

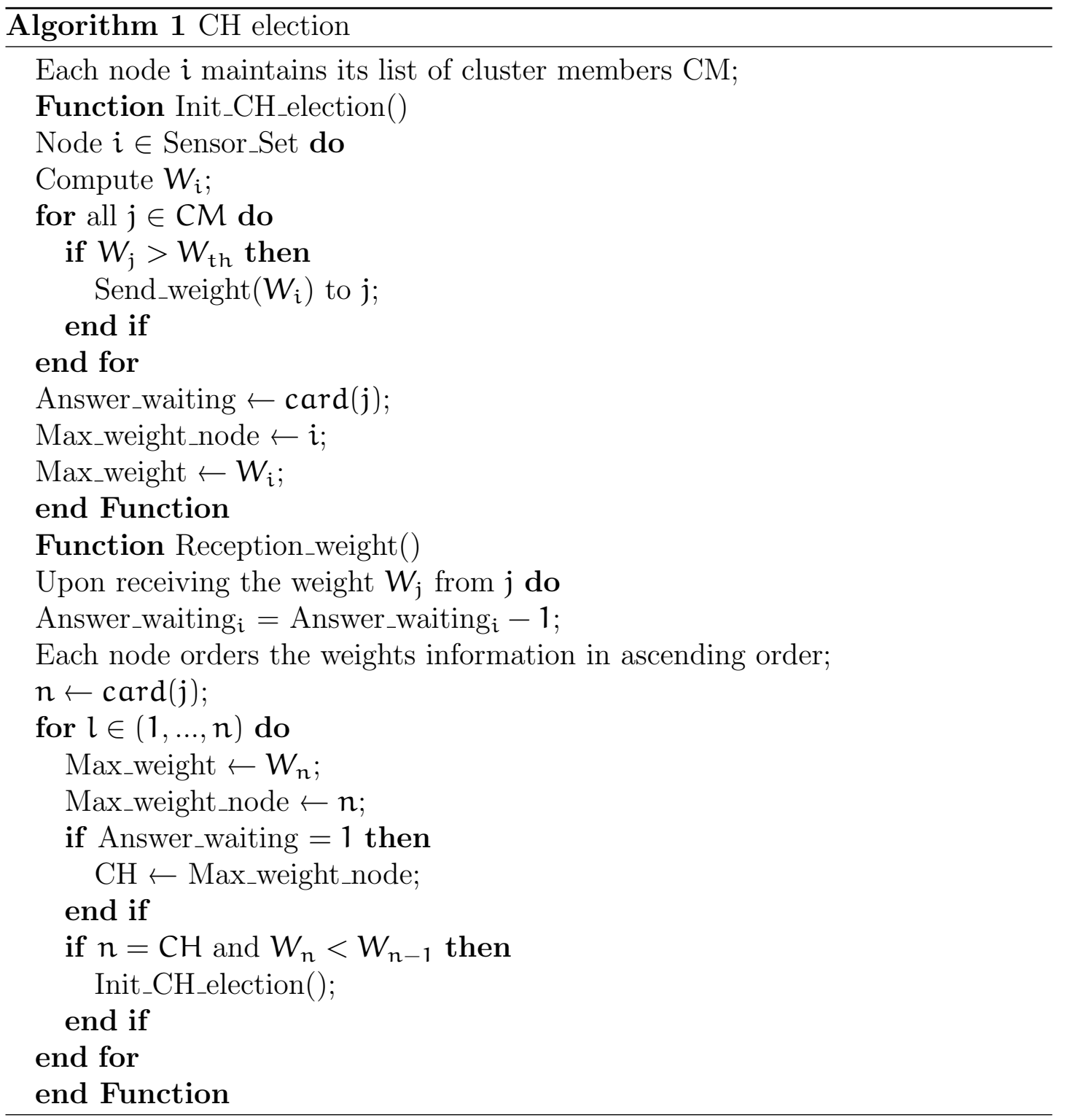

\subsection{Delay-energy aware routing algorithm}

We model the network with a graph $G(V, E)$, where $V$ denotes the network nodes and $E$ represents the communication links. When an event is triggered by a sensor node, the corresponding $\mathrm{CH}$ should identify the most efficient routing path reaching an actuator node $A$. Our algorithm follows an on-demand routing 
approach [22]. The protocol establishes the routing paths to the actuator node $A$ only when the $\mathrm{CH}$ needs to send an alert. It establishes the latter routing paths according to the intermediate sensor nodes energy and communication delay. To discover the routing paths towards the actuator node $A$, the $\mathrm{CH}$ initiates the route recovery with the creation of a route request packet RREQ. The latter is forwarded through the intermediate sensor nodes until the actuator node A. Each intermediate sensor node embeds both information related to the transmission delay and the energy state in the RREP packet. As there are more than one intermediate sensor node, multiple RREP packets may be received by the $\mathrm{CH}$. In this case, the $\mathrm{CH}$ can make a decision to select the best routing path. In Figure 4. we illustrate an example of the route discovery process executed between a $\mathrm{CH}$ and an actuator node $A$.

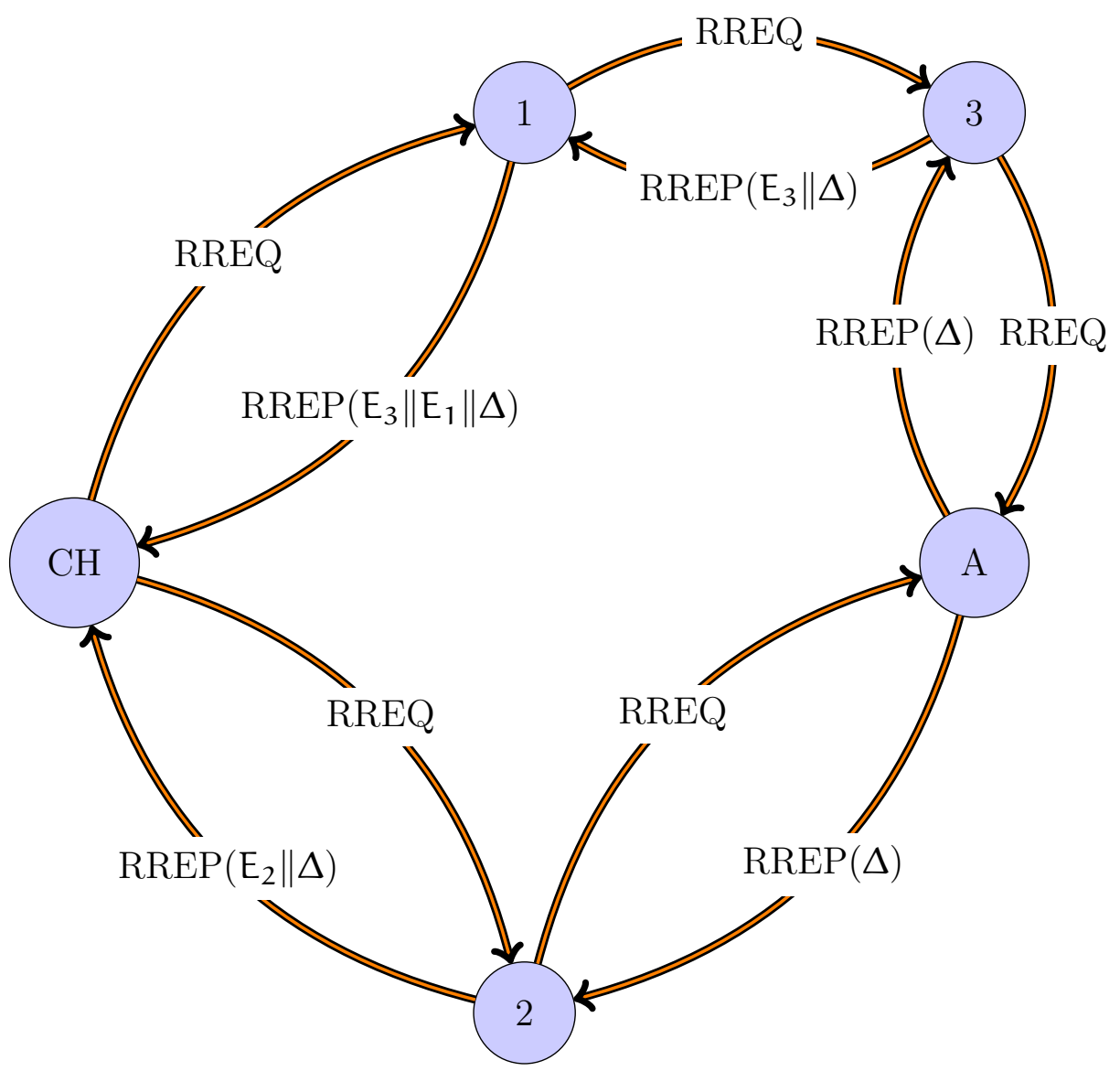

Figure 4: The process of creation of RREQ and RREP packets ( $E_{i}$ denotes the residual energy of the node $i$ and $\Delta$ the communication delay)

The existence of multiple paths between the pair of vertices, the source node $\mathrm{CH} \in \mathrm{V}$ and the destination node $\mathrm{A} \in \mathrm{V}$, provides the possibility of some routing 
paths being better than others in terms of their associated costs. The $\mathrm{CH}$ selects the best routing path $\mathrm{P}^{*}\langle\mathrm{CH}, \mathrm{A}\rangle$ having the optimal cost such as

$$
\mathrm{C}\left(\mathrm{P}^{*}\langle\mathrm{CH}, \mathrm{A}\rangle\right)=\max \left(\mathrm{C}\left(\mathrm{P}_{1}\langle\mathrm{CH}, \mathrm{A}\rangle\right), \mathrm{C}\left(\mathrm{P}_{2}\langle\mathrm{CH}, \mathrm{A}\rangle\right), \cdots, \mathrm{C}\left(\mathrm{P}_{\ell}\langle\mathrm{CH}, \mathrm{A}\rangle\right)\right)
$$

where $\ell$ is the existing routing path number between the $\mathrm{CH}$ and the actuator node $A$, and

$$
\mathrm{C}\left(\mathrm{P}_{\mathrm{i}}\langle\mathrm{CH}, \mathrm{A}\rangle\right)=\frac{\mathrm{R}\left(\mathrm{P}_{\mathrm{i}}\langle\mathrm{CH}, \mathrm{A}\rangle\right)}{\triangle\left(\mathrm{P}_{\mathrm{i}}\langle\mathrm{CH}, \mathrm{A}\rangle\right)},
$$

where $R(P\langle C H, A\rangle)$ represents the average residual energy of the intermediate sensor nodes between the $\mathrm{CH}$ and the actuator node $A$ and $\triangle(\mathrm{P}\langle\mathrm{CH}, \mathrm{A}\rangle)$ represents the delay experienced on this path.

In our solution, the actuator node responds to the received RREQs following the specifications of on-demand routing protocol. However, in certain inherent situations, an actuator node may be not able to respond positively to a request packet, i.e., it is unable to perform immediately the requested action. This could happen if the actuator node is not able to act in the event area, when it occurs for example in a geographically inaccessible region. Moreover, the actuator node may be busy in reacting to other events or its actual energy state does not allow the execution of the requested action. In such cases, the actuator node sends a negative acknowledgement to the $\mathrm{CH}$. Finally, the latter should consider another actuator node in order to react to the detected event since the $\mathrm{CH}$ position is approximately in the center of actuator nodes. We notice that it is quite rare that an actuator node does not respond completely to received RREQs either by a positive or negative acknowledgement. This may happen if the actuator node is out of the network connection, completely failed, or due to an excessive concentration of the communication load in the environment. In such cases, our solution acts identically as the basic version of on-demand routing protocol.

\section{Performance evaluation}

In this section, we present the simulation parameters, and then the obtained results in terms of clustering and routing. 


\subsection{Simulation environment and metrics}

In order to analyze the performances of our approach we have performed simulations, which we have implemented ${ }^{1}$ using the MATLAB environment. We have not provided a real implementation and/or a case study. We believe that the simulation study is more appropriate regarding the metrics to evaluate (scalability, event intensity, etc.). The environment setting would be hard to realize by real experiments when evaluating such metrics, due to the high size of network, the high number of randomly network deployment scenarios and generated events. In addition, several important probabilistic characteristics are difficult to schedule by real experiments, such as the message loss and the channel collisions. Furthermore, we have taken in charge real measures in order to bring the results closer to the real case.

We evaluate the performances of our approach with comparison to two protocols. Indeed, we propose a framework of data communication composed of two complement solutions. The first solution is about clustering. This approach consists to cluster efficiently the network in order to allow reliable access to the actuators. We compare this part of the proposed framework to the clustering approach CCR [16]. The second solution that we propose is about routing. The routing aspect is addressed independently to the clustering, where is assumed that the source node is the $\mathrm{CH}$ and the destination node is the targeted actuator. This approach allows reaching the actuator node with a minimum delay and a reduced energy consumption. We compare this part of the proposed framework to the routing approach KanGuRou [23]. In terms of clustering, we notice that the CCR protocol is the most representative solution for comparison. Unlike the nature of our solution, those ones proposed in [3, [7] and [9] elect the actuator nodes as CHs, which is unsuitable regarding the cost of deployment in large scale. For a great network size, the number of actuators to deploy is large. In addition, the solutions proposed in [8] and [14] do not consider one of the most important metric, which is the degree of connectivity of the candidate sensor nodes. In terms of routing, we believe that the KanGuRou protocol is the most suitable for comparison. In this protocol, the problem of actuator nodes accessibility is directly addressed, which is one of the main purposes of the proposed framework, i.e., each sensor can reach at least one actuator node.

We simulate a WSAN with stationary sensor nodes, which are deployed randomly on the surveillance field, and stationary actuator nodes distributed uniformly in the same area. In WASNs, a high number of sensors have to be deployed.

\footnotetext{
${ }^{1}$ The developed simulator is a project part supported by the laboratory LIMED consisting of the implementation of personalized simulator libraries. Project title: "Security and optimization in surveillance and support networks - modeling and applications", project code: "B*00620140067", University of Bejaia, Algeria.
} 
In order to evaluate the scalability criterion, which is a crucial metric in such networks, we have considered a network size varied between 20 and 400 sensor nodes and up to 12 actuators. In the simulations, we have considered the message loss rate denoted by $\alpha$. Indeed, WASNs are a special case of LLNs (Low-power and Lossy Networks), where the deployment of the network is in most of the cases for hostile environments. The nature of this type of environments has a direct impact on the radio transmission quality (obstacles, noise, temperature, interferences with the observed system, etc.). To address this important characteristic, the message loss rate $\alpha$ is set to $20 \%$, which is common for all the compared solutions. In addition, we have considered the collision probability, denoted by $\beta$ and computed such as

$$
\beta=1-\frac{1}{s+1},
$$

where $s$ represents the average neighbor number per node. The parameter $\beta$ is proportional to $s$, where the probability of collision increases in function of the neighboring density.

We have considered the frequency of $\mathrm{CH}$ algorithm refresh. The beacon messages are exchanged periodically at each period of time $\gamma$ between the cluster members in order to establish and maintain the $\mathrm{CH}$ election. We set $\gamma=5 \mathrm{mnt}$, and later, we vary this metric in order to study its impact. Since the targeted applications in the scope of this paper are time-sensitive, we have given priority to the delay criterion, where the coefficient $\mathrm{c}$ is set to 0.4 . The action performed by the actuator nodes is mechanical in most of the application cases. Therefore, they spend much energy when they perform the operation. In the simulations, the energy consumed by an actuator for an action is set to 5\%. Finally, a timeout is introduced for the lack of actuator responses, which we have set to 5 s for all the simulation scenarios. In Table 2, we summarize the main simulation parameters.

\subsection{Clustering approach}

Due to the limitation of energy of sensor and actuator nodes, it is important that the energy be efficiently used. The energy consumption of our approach is compared to CCR as shown in Figure 5. The average residual energy of the sensor nodes is greater in our approach compared to CCR. In our approach, the $\mathrm{CH}$ is closest to the actuator nodes, which provides minimum energy consumption. Also, the average residual energy of actuator nodes is greater in our protocol compared to CCR as illustrated in Figure6. In CCR, if an actuator node can not respond to a request forwarded by the $\mathrm{CH}$, it coordinates with other actuator nodes, which provides high energy consumption. However, in our approach the $\mathrm{CH}$ sends the request directly to the second best actuator node. Figure 7 illustrates the average energy consumption in the network in function of the number of sensor nodes. 


\begin{tabular}{|l|l|}
\hline Parameter & Value \\
\hline Surface of interest size & $100 \times 100 \mathrm{~m}^{2}$ \\
Number of sensor nodes & 20 to 400 \\
Number of actuator nodes & 12 \\
Sensor nodes deployment & Random \\
Actuator nodes deployment & Uniform \\
Sensor node radio range & $20 \mathrm{~m}$ \\
Actuator node radio range & $40 \mathrm{~m}$ \\
Packet size & 56 Bytes \\
Event number & 10 to 80 \\
Initial energy of sensor nodes & $1 \mathrm{Joule}$ \\
Initial energy of actuator nodes & $4 \mathrm{Joule}$ \\
Coefficient c & 0.4 \\
Message loss rate $\alpha$ & $20 \%$ \\
Frequency of CH refresh $\gamma$ & 5 to $25 \mathrm{mnt}$ \\
Timeout & $5 \mathrm{~s}$ \\
Energy consumed by an actuator for an action & $5 \%$ \\
\hline
\end{tabular}

Table 2: Simulation parameters

The energy consumption in our approach is much lower than CCR. In CCR, the choice of the $\mathrm{CH}$ depends only on the residual energy and the nodes density. Figure 8 shows that our approach provides lower packet delay to send the data from the $\mathrm{CH}$ toward the actuator nodes compared to CCR. Figure 9 shows that our approach provides also lower event reporting delay than CCR. The obtained results indicate that our approach reduces the communication delay in the network due to the consideration of all the actuator nodes in the $\mathrm{CH}$ election.

Figure 10 presents the intersection of the average residual energy and the maximal value of energy consumed by sensor nodes for different scenarios of event frequency occurring. We note that the value of the $\mathrm{CH}$ refresh interval $\gamma$ influences highly on the residual energy of sensor nodes. Indeed, the optimal value of $\gamma$ depends on several parameters relating to the network and the environment. Furthermore, this value increases by increasing the frequency of events occurring. For example, in the case of a frequency of one event per minute, the optimal value to consider by the application is about $\gamma=8 \mathrm{mnt}$.

\subsection{Routing approach}

The energy consumption of our routing approach is compared to the KanGuRou protocol and the results are illustrated in Figure 11, Figure 12 and Figure 13. The energy consumption with our approach is lower than KanGuRou. When the number of sensor and actuator nodes increases, the efficiency of our approach 


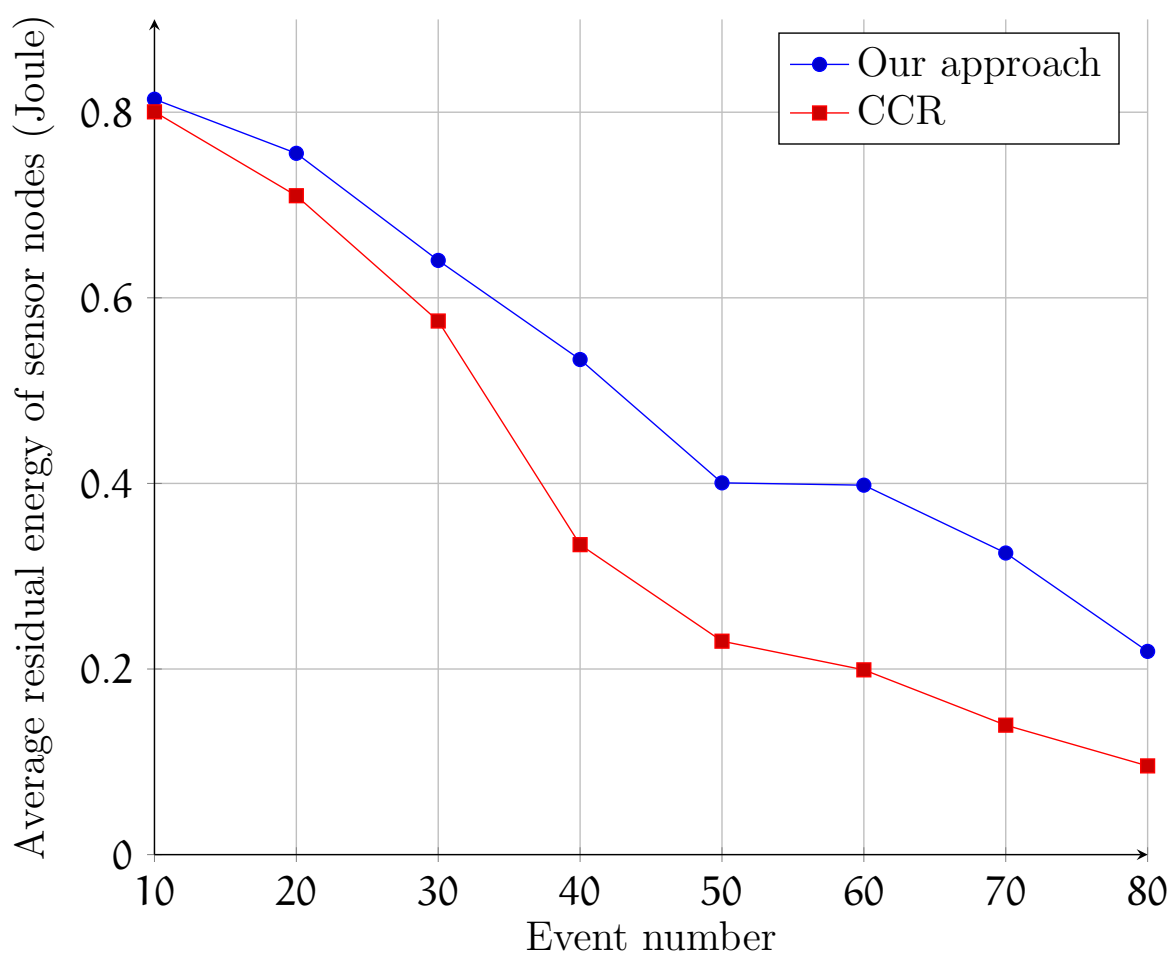

Figure 5: Average residual energy of sensor nodes in function of the event number

is more significant than KanGuRou. The obtained results show that our approach is better scalable and enables energy saving due to the data delivery path, which is shorter, and hence, fewer transmissions per path are required. Figure 14 and 15 illustrate the communication delay in the network in function of the sensor node number. As expected, the communication delay in KanGuRou and our approach increases when the network size increases. However, the communication delay is significantly less in our protocol because the data is forwarded using the optimal path compared to KanGuRou, which diffuses the packet over several routing paths.

\section{Conclusions and future works}

In this paper, we have proposed an energy efficient and QoS aware routing protocol for WSANs. We have proposed a new clustering mechanism that takes into consideration the accessibility degree of the actuator nodes in the process of $\mathrm{CH}$ election. Our approach combines two other important parameters, namely the energy state and the degree of connectivity of the candidate sensor nodes. Our approach reduces the energy consumption as well as the communication delay and balances the energy consumption equally among the actuator nodes. This 


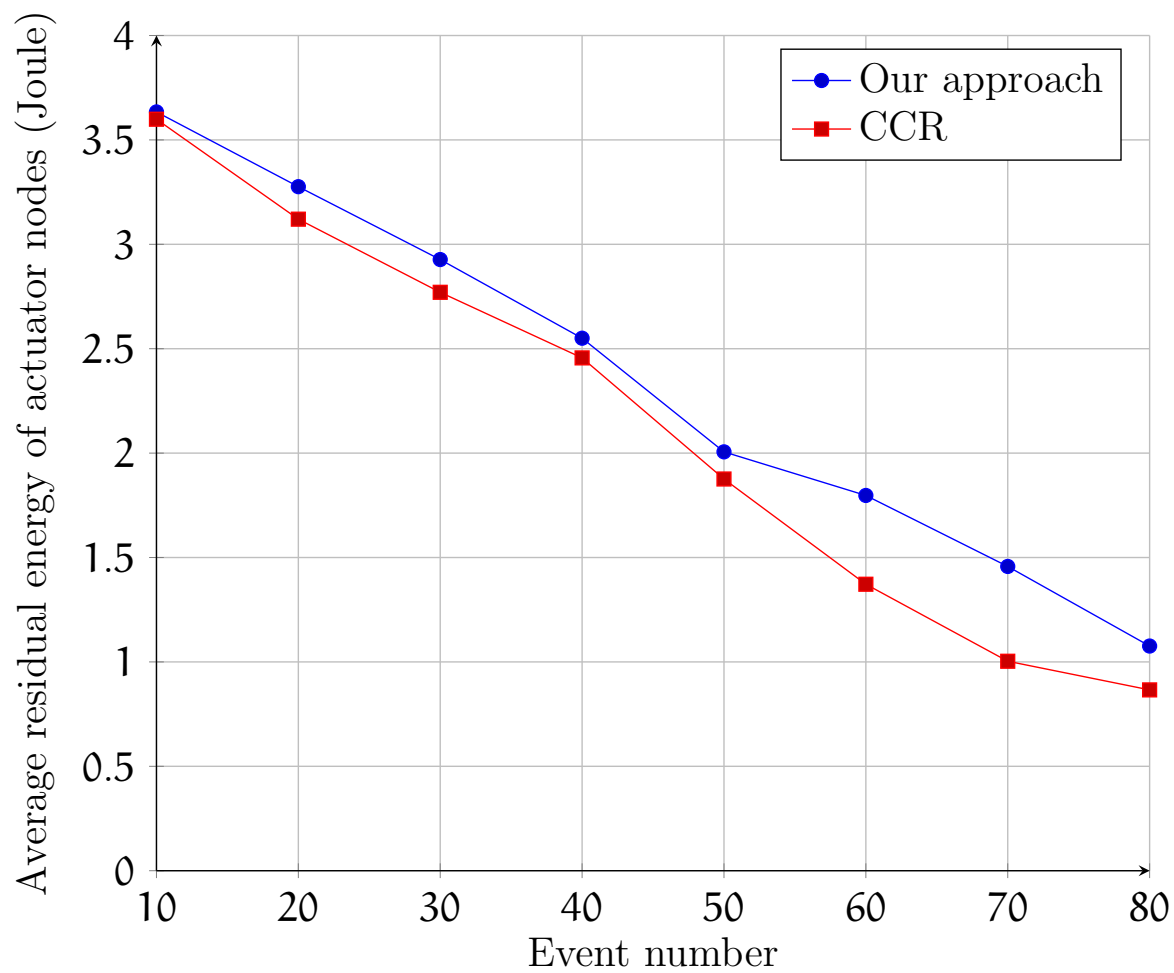

Figure 6: Average residual energy of actuator nodes in function of the event number (6 actuators)

is due to the propriety that the candidate sensor node position should be in the best at the center of the actuator nodes locations. We have also addressed the sensor-actuator communication and we have proposed an on-demand routingbased data communication protocol, which allows reaching the actuator node with a minimum delay and a reduced energy consumption. The simulation results show the performance of our framework. The energy of both sensor and actuator nodes are efficiently used with lightweight communication delay, which leads to a longer network lifetime.

The proposed framework is suitable for a panoply of applications. For instance, it can be deployed for fire sprinkler systems monitoring, which are in charge to detect, control, suppress and extinguish fires. Whether a sensor node detects an event, a water sprinkler actuator is remotely notified in order discharge water in short delay before the fire be propagated. The Supervisory Control and Data Acquisition (SCADA) system is another type of application, which can deploy the proposed framework. SCADA refers to computer systems that monitor and control industrial processes, in which the sensors and actuators can be used for factory automation, inventory management, and detection of liquid and gas leakages. The proposed framework can also be deployed in other sensitive-delay 


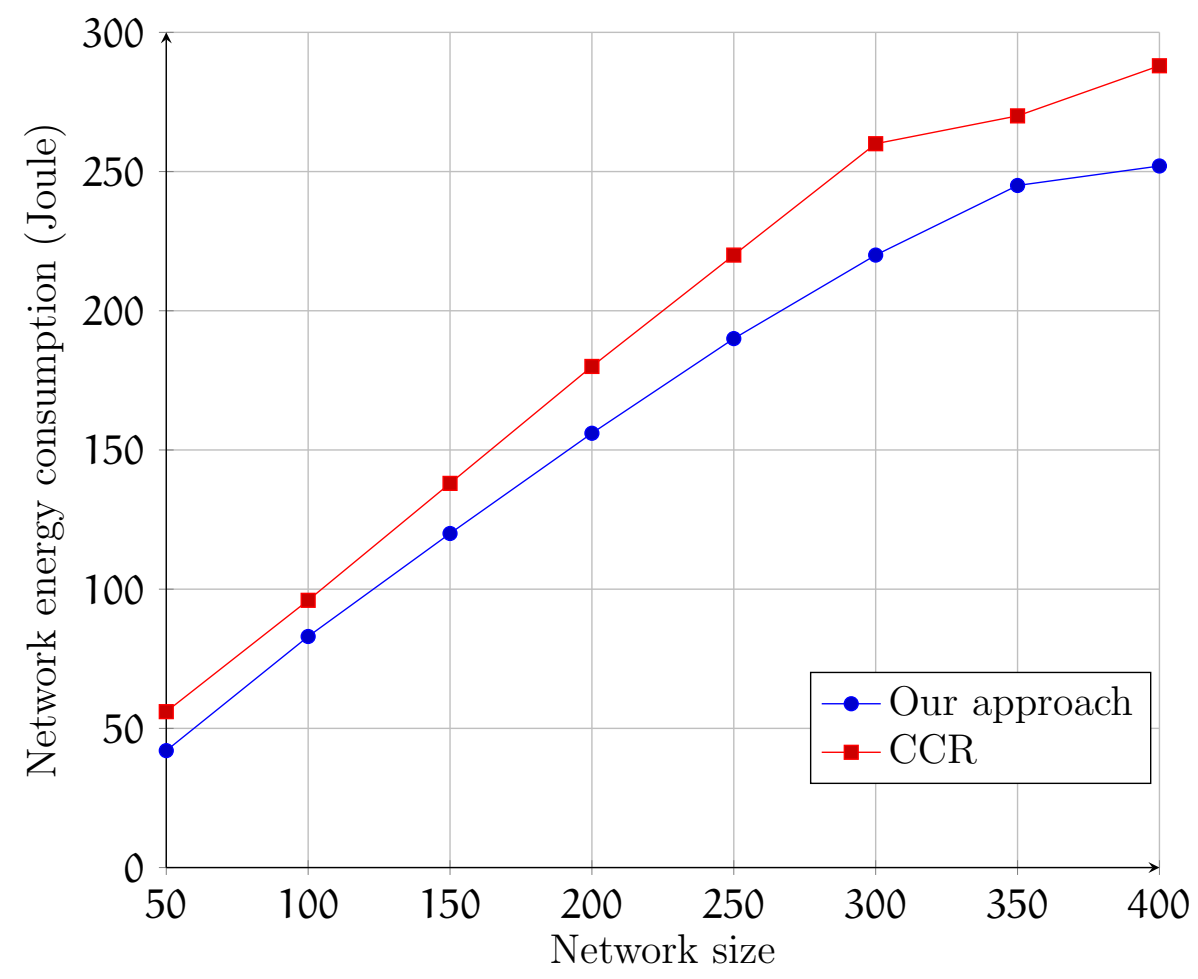

Figure 7: Energy consumption in function of the network size under 70 events

applications, in which strict delays must be ensured so that the elapsed time between the detection of an anomaly and the intervention of the operator enables the resolution of the incident.

As medium-term prospects, we are planning to adapt the proposed framework in the context of mobile WSANs. A fully distributed solution based on actuator tracking is being designed in order to guess the actuator positions and reach them efficiently when an event is triggered. As long-term prospects, we plan to investigate the security aspects of such networks. Lightweight security solutions should be designed to address several types of attacks, such as eavesdropping, identity usurpation, black-hole, jamming, etc.

\section{Acknowledgment}

This work was carried out in the framework of research activities of the laboratory LIMED, which is affiliated to the Faculty of Exact Sciences of the University of Bejaia. It was done in collaboration with the Labex MS2T, which was funded by the French Government, through the program "Investments for the future" managed by the National Agency for Research (Reference ANR-11-IDEX-000402). 


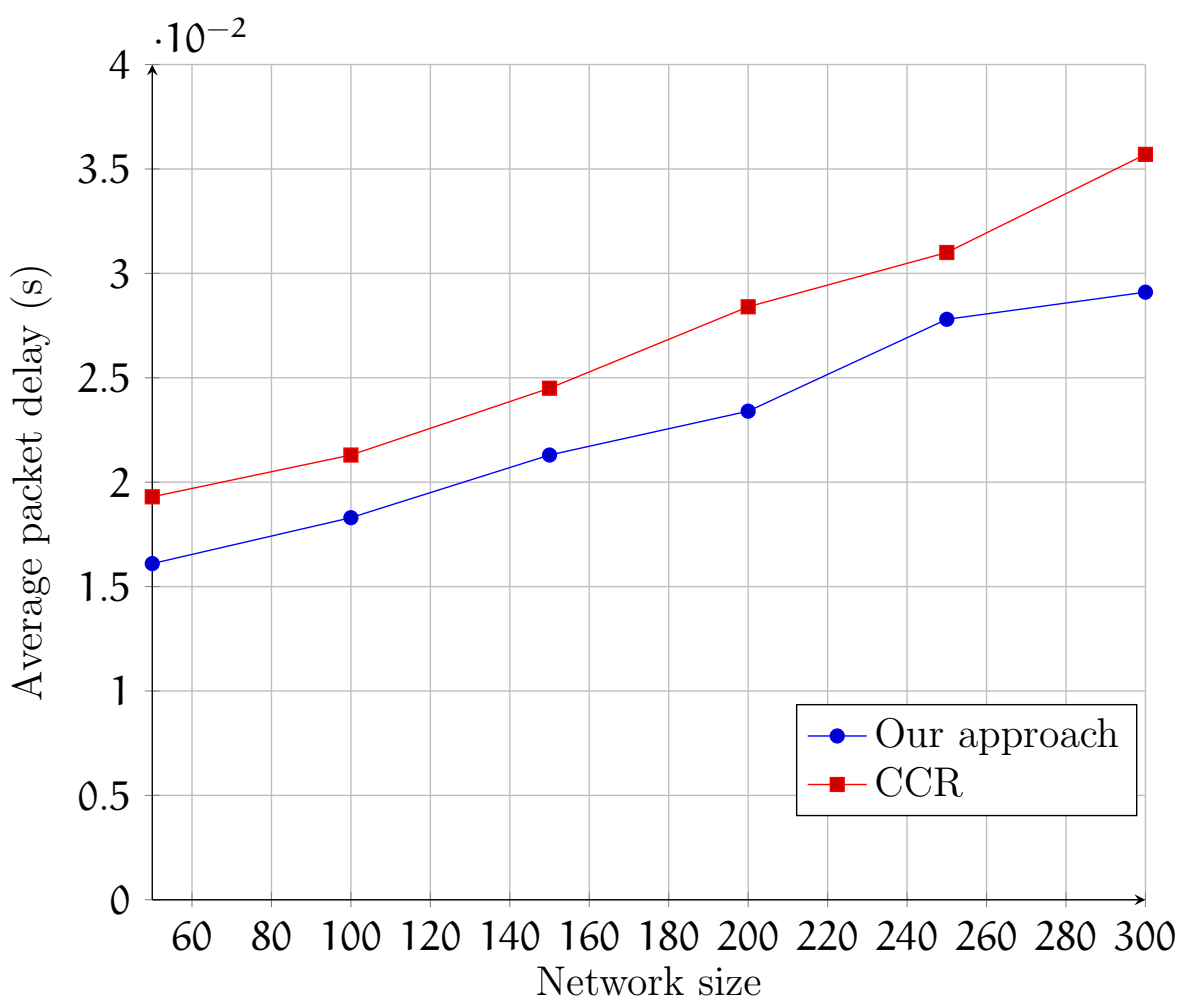

Figure 8: Packet delay between $\mathrm{CHs}$ and actuator nodes in function of network size under 10 events

\section{References}

[1] Salarian H, Chin KW, Naghdy F. Coordination in wireless sensor actuator networks: a survey. Journal of Parallel and Distributed Computing July 2012; 72(7):856-867.

[2] Akbas MI, Turgut D. Lightweight routing with QoS support in wireless sensor and actor networks. In Proceeding of the IEEE GLOBECOM 2010.

[3] Zeng Y, Sreenan CJ, Zheng G. A real-time architecture for automated wireless sensor and actuator networks. In Proceedings of the 5th International Conference on Wireless and Mobile Communications August 2009.

[4] Boukerche A, Araujo RB, Villa L. A novel QoS based routing protocol for wireless actor and sensor networks. In Proceeding of the IEEE GLOBECOM November 2007; 4931-4935.

[5] Canete E, Diaz M, Liopis L, Rubio B. HERO: a hierarchical, efficient and reliable routing protocol for wireless sensor and actor networks. Computer Communications 2012; 35:1392-1409. 


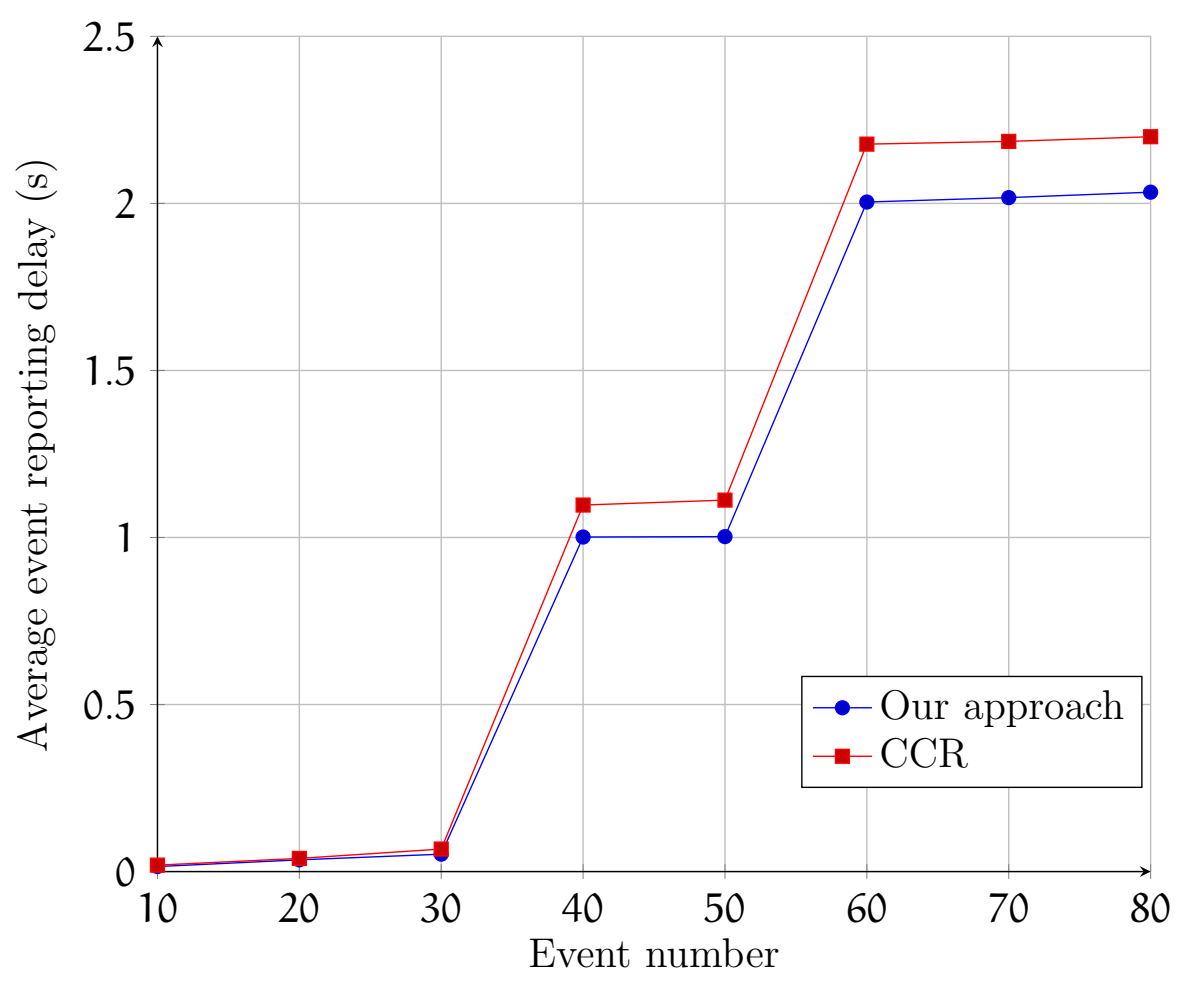

Figure 9: Reporting event delay in function of the event number

[6] Ngai ECH, Lyu MR, Liu J. A real-time communication framework for wireless sensor-actuator networks. In Proceeding of the IEEE Aerospace Conference 2006.

[7] Melodia T, Pompili D, Gungor VC, Akyildiz I. F. A distributed coordination framework for wireless sensor and actor networks. In Proceeding of MobiHoc'05 May 2005.

[8] Khan MA, Shah GA, Sher M . A QoS based multicast communication framework for wireless sensor actor networks (WSANs). ICIC international journal December 2011; 7(12):7003-7020.

[9] Xiang K, Zeng Y, Sun B. QoS guaranteed architecture for wireless sensor and actuator networks, IEEE WiCOM September 2010.

[10] Durresi A, Paruchri V, Barolli L. Delay-energy aware routing protocol for sensor and actor networks. In proceedings of ICPADS'05 2005.

[11] Ngai E, Zhou Y, Lyu MR, Liu J. A delay-aware reliable event reporting framework for wireless sensor-actuator networks. Ad Hoc Networks September 2010; 8(7):694-707. 


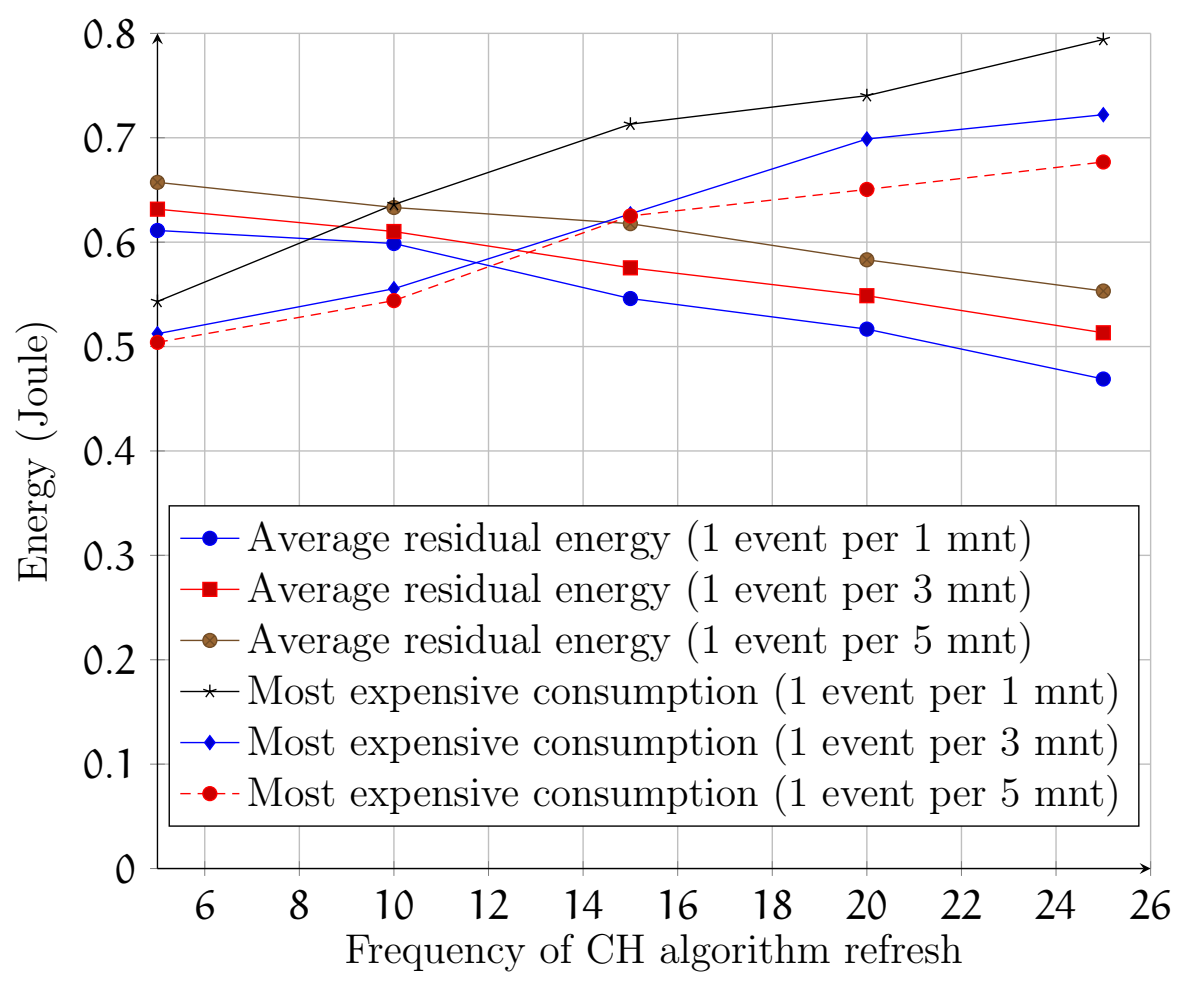

Figure 10: Cost of the frequency of $\mathrm{CH}$ algorithm refresh over the frequency of events

[12] Zeng Y, Li D, Vasilakos AV. A real-time data report and task execution in wireless sensor-actuator networks using self-aware mobile actuators. Computer Communications August 2012; 988-997.

[13] Khan MA, Shah GA, Ahsan M, Sher M. An efficient and reliable clustering algorithm for wireless sensor actor networks (WSANs). 53rd International Midwest Symposium on Circuits and Systems (MWSCAS) August 2010; 332338 .

[14] Gungor VC, Sastry C, Song Z, Integlia R. Ressource-aware and link quality based routing metric for wireless sensor and actor networks. In Proceedings of ICC'07 June 2007; 3364-3369.

[15] Akyildiz If, Kasimoglu IH. Wireless sensor and actor networks: research challenge. Ad Hoc Networks 2004; 2(4):351-367.

[16] Shah GA, Bozyigit M, Hussain FB. Cluster-based coordination and routing framework for wireless sensor and actor networks. Wireless Communications and Mobile Computing December 2009; 1140-1154. 


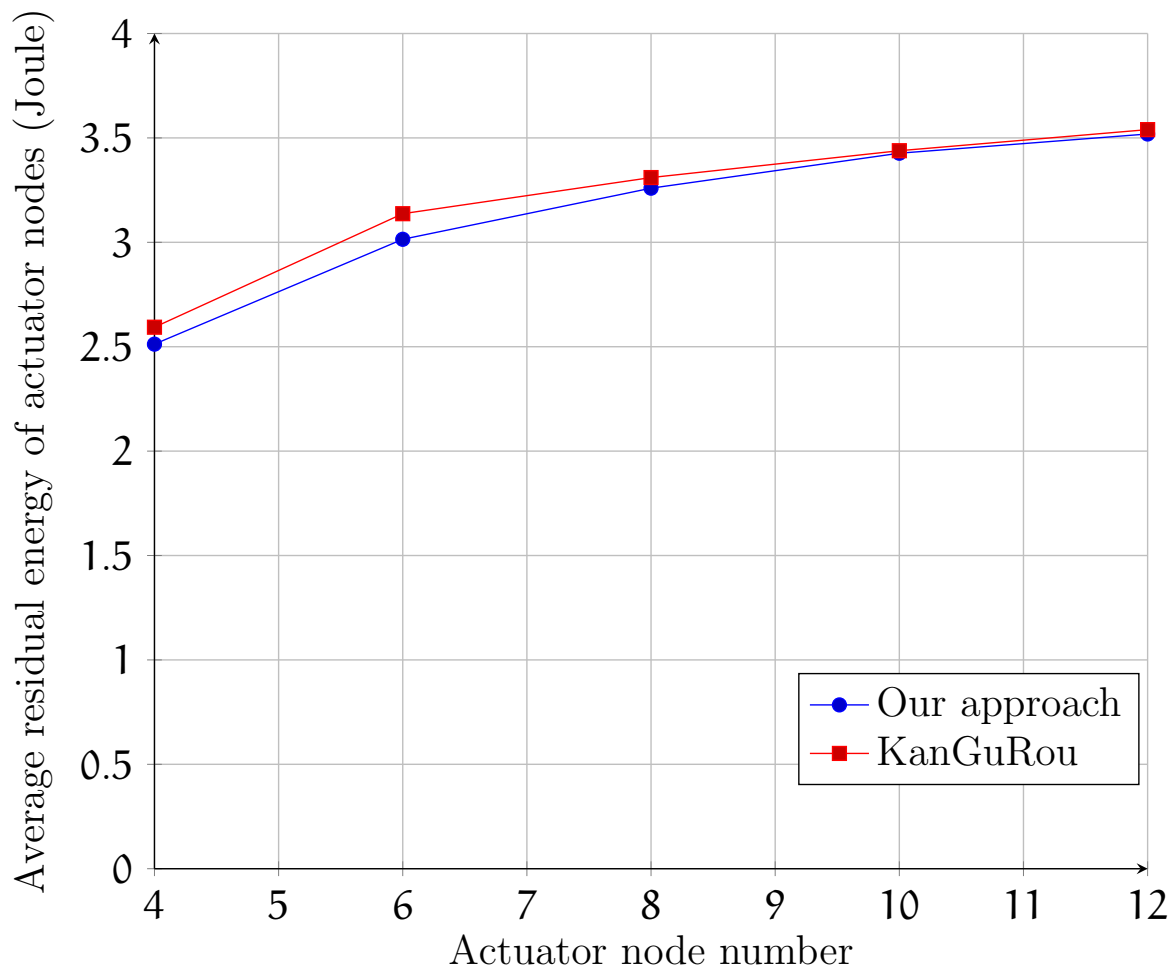

Figure 11: Energy consumption of actuator nodes in function of the actuator number ( 50 sensor nodes, 30 events)

[17] Verdone R, Daradri, Mazzini G, Conti A. Wireless sensor and actuator networks: technologies, analysis and design. Academic Press 2008.

[18] Wark T, Crossman C, Hu W, Guo Y, Valencia P, Corke P, Lee C, Henshall J, Prayaga K, Grady JO, Reed M, Fisher A. The design and evaluation of a mobile sensor/actuator network for autonomous animal control. In Proceedings of the 6th International Conference on Information Processing in Sensor Networks April 2007.

[19] Akkaya K, Younis M. A survey on routing protocols for wireless sensor networks. Ad Hoc Networks'3 November 2003.

[20] Anastasi G, Conti M, Francesco MD, Passarella A. Energy conservation in wireless sensor networks: a survey. Ad Hoc Networks July 2008.

[21] Demirkol I, Ersoy C, Alagoz F. MAC protocols for wireless sensor networks: a survey. IEEE Communications Magazine April 2006.

[22] Perkins CE, Royer EM. Ad Hoc On-Demand Distance Vector Routing. In 


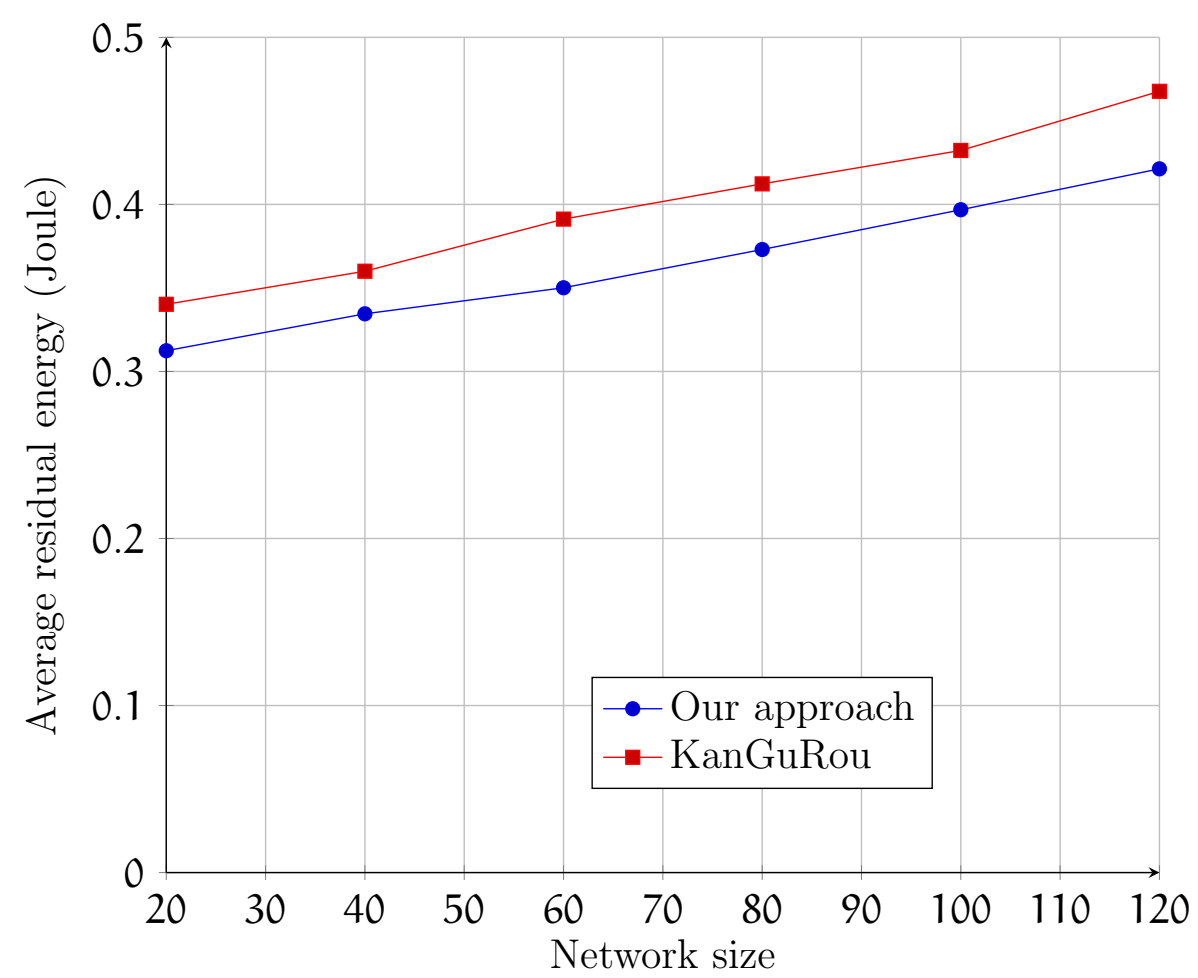

Figure 12: Energy consumption of sensor nodes in function of the network size with 6 actuator nodes

Proceedings of the 2nd IEEE Workshop on Mobile Computing Syslems and Applications February 1999; 90-100.

[23] Mitton N, Ryl DS, Voge ME, Zhang L. Energy Efficient k-Anycast Routing in Multi-sink Wireless Networks with Guaranteed Delivery. 11th International Conference on Ad-Hoc Networks and Wireless 2012; 385-398.

[24] Dinh T, Kim Y. Actor-oriented directional anycast routing in wireless sensor and actor networks with smart antennas. Wireless Networks 2016.

[25] Li B, Ma Y, Westenbroek T, Wu C, Gonzalez H, Lu C. Wireless Routing and Control: a Cyber-Physical Case Study. Wireless Networks. ACM/IEEE 7th International Conference on Cyber-Physical Systems 2016.

[26] Long J, Gao C, He S, Liu X, Liu A. Bridging the gap among actorsensoractor communication through load balancing multi-path routing. Journal on Wireless Communications and Networking 2015; 1-22.

[27] Kumar A, Ranga V. A Cluster-Based Coordination and Communication 


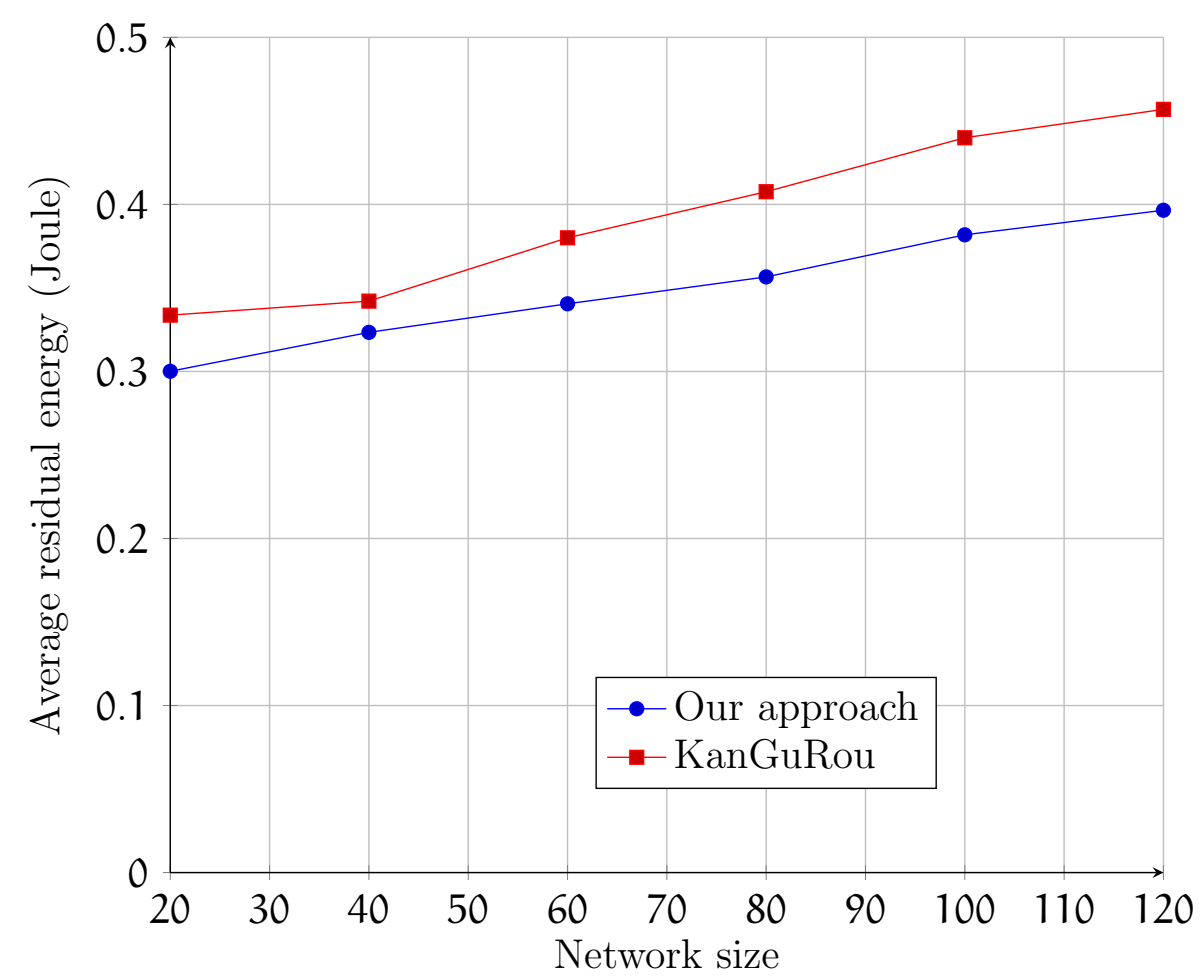

Figure 13: Energy consumption of sensor nodes in function of the network size with 8 actuator nodes

Framework Using GA for WSANs. Proceedings of Ninth International Conference on Wireless Communication and Sensor Networks April 2014; 111-124.

[28] Sanap M. E and Satao R. A. "Bin SDR": Effective Algorithm for Wireless SensorActor Network. Intelligent Computing, Networking, and Informatics, Advances in Intelligent Systems and Computing 243 2014; 389-395.

[29] Quang P. T. A, Kim D. S. Clustering Algorithm of Hierarchical Structures in Large-Scale Wireless Sensor and Actuator Networks. Journal of communications and networks October 2015; 17(5):473-481.

[30] Kakarla J, Majhi B, Battula R. A delay and energy aware coordination mechanism for WSAN. International journal of communication systems October 2016.

[31] Shokouhifar M, Jalali A. A new evolutionary based application specific routing protocol for clustered wireless sensor networks. AEU-Int J Electron Commun 2015;69(1):432-441. 


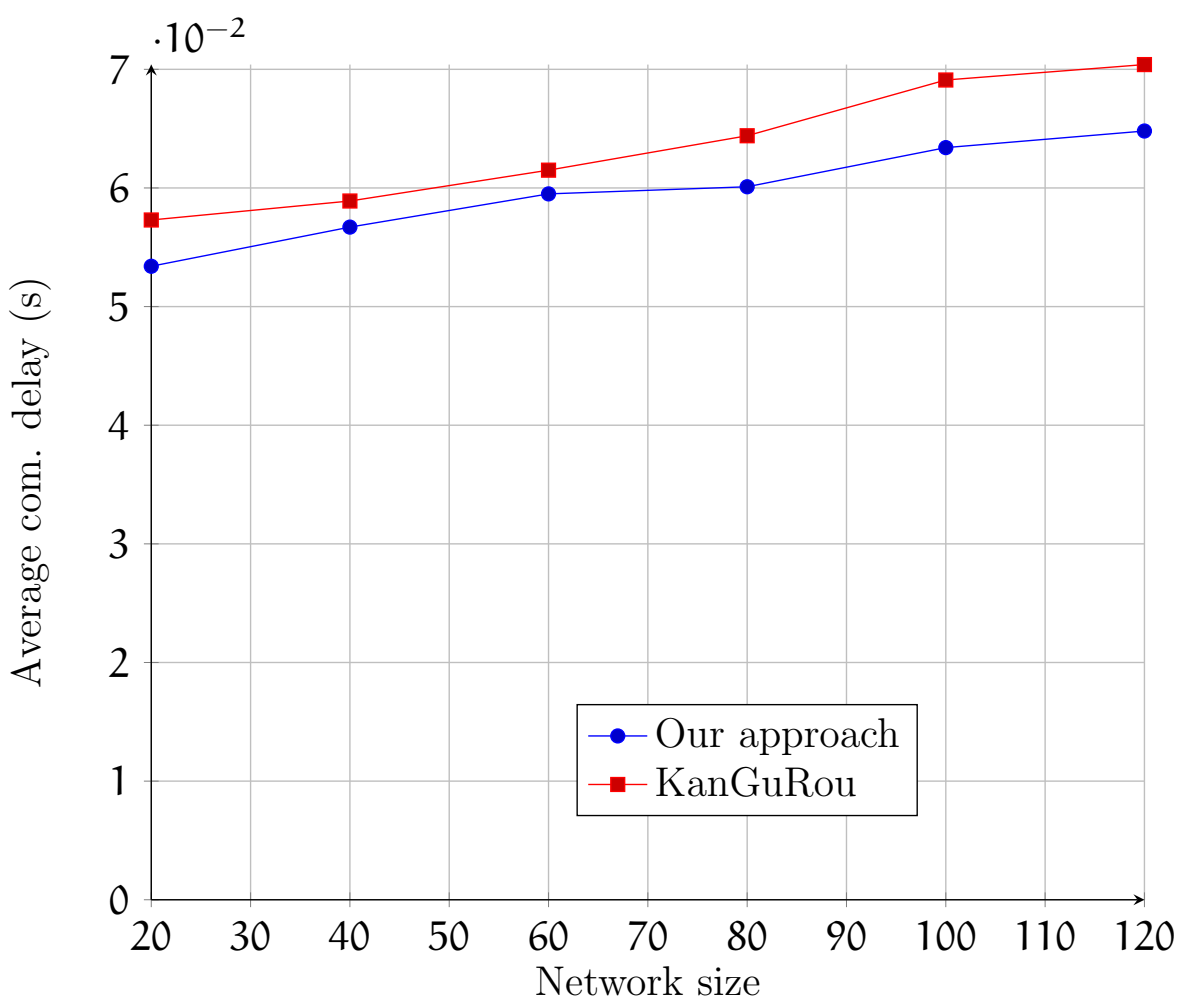

Figure 14: Communication delay in function of the network size with 6 actuator nodes

[32] Maryam S, Reza NH. A decentralized energy efficient hierarchical clusterbased routing algorithm for wireless sensor networks. AEU-Int J Electron Commun 2015;69(5):790-799.

[33] Yu J, Qia Y, Wangb G, Gua X. A cluster-based routing protocol for wireless sensor networks with nonuniform node distribution. AEU-Int J Electron Commun 2012;66:54-61.

[34] Boukerche A, Araujo R.B, Silva F.H.S, Villas L. Wireless sensor and actor networks context interpretation for the emergency preparedness class of applications. Computer Communications 2007; 30: 2593-2602.

[35] Norseth K. C, Romascanu D, Bierman A. Entity Sensor Management Information Base. IETF ROLL, RFC 3433, 2002.

[36] Alexander R, Brandt A, Vasseur J.P, Hui J, Pister K, Thubert P, Levis P, Struik R, Kelsey R, Winter T. RPL: IPv6 Routing Protocol for Low-Power and Lossy Networks. IETF ROLL, RFC 6550, 2012. 


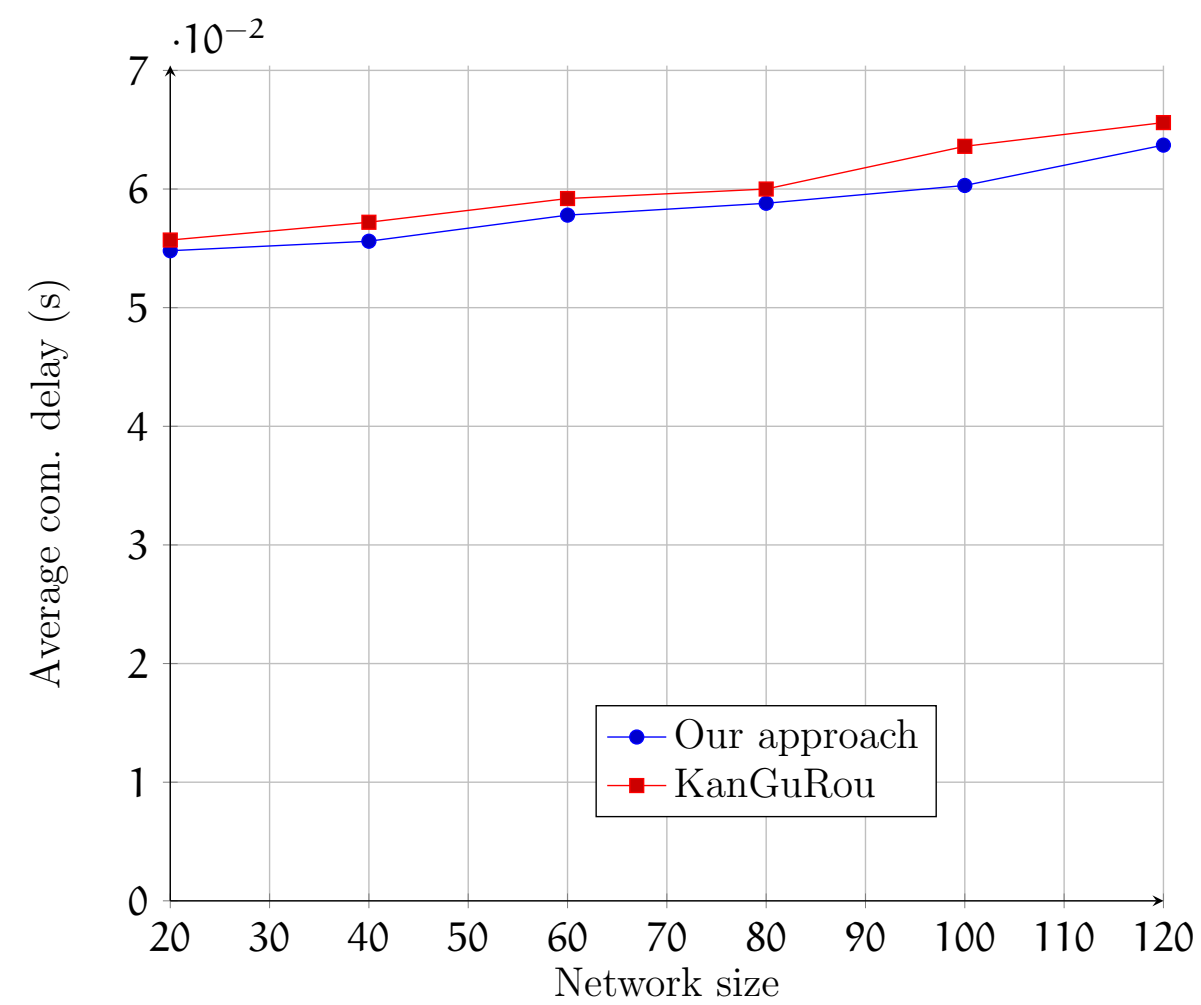

Figure 15: Communication delay in function of the network size with 8 actuator nodes 